\title{
La tutela del minore e il diritto di visita del genitore in caso di sottrazione internazionale
}

\author{
Marta Rovacchi
}

\section{Riassunto}

L'Autore affronta il tema della sottrazione internazionale dei minori e del diritto di visita del genitore in caso di sottrazione internazionale.

Al fine di valutare se il trasferimento all'estero del minore o il suo mancato rientro è illecito occorre muovere dall'art. 3 della Convenzione dell'Aja.

Occorre poi distinguere - come spiega l'Autore - la fattispecie della sottrazione internazionale del minore dal trasferimento all'estero del genitore in presenza di determinate circostanze dettate dal giudice in punto all'affidamento.

L'Autore indica infine i rimedi a disposizione del genitore al quale il minore è sottratto illecitamente a causa del suo trasferimento all'estero ad opera dell'altro.

\section{Résumé}

L'auteur aborde la question de l'enlèvement international d'enfants et du droit de visite du parent.

Afin de déterminer si le déplacement du mineur à l'étranger, ou si son non-retour, est illicite, il est nécessaire de se baser sur l'article $\mathrm{n}^{\circ} 3$ de la Convention de La Haye.

Comme l'explique l'auteur lui-même dans son texte, il est nécessaire de distinguer entre le cas de l'enlèvement international du mineur et celui du déplacement international de l'enfant par l'un de ses parents dans des circonstances prévues par le juge dans son jugement sur la garde de cet enfant.

Enfin, l'auteur indique les mesures à la disposition du parent auquel le mineur a été enlevé illicitement par l'autre parent à cause de son déplacement à l'étranger.

\section{Abstract}

The author deals with the issue of the international child abduction and the parental visitation rights in case of international child abduction.

In order to assess if the child's transfer to another country or their retention (wrongful removal) is legally carried out it is necessary to refer to art.3 of Aja Convention.

Then, as the author explains, it is necessary to distinguish the case concerning the international child abduction from the case concerning the parental transfer to another country because of particular circumstances ordered by the Judge at the moment of the child custody.

At last the author indicates the possible solutions adoptable by the parent whose child has been illegally abducted by the other parent who has transferred the child's residence abroad.

Key words: child protection; Aja Convention; parental visitation rights; international child abduction; parental transfer to another country.

\footnotetext{
- E' iscritta all'Albo degli Avvocati presso l'Ordine di Reggio Emilia dal 1993. Associata AIAF (Associazione Italiana Avvocati per la Famiglia e per i Minori), è membro del Direttivo Nazionale e di quello Regionale, oltre ad essere la responsabile della sezione territoriale di Reggio Emilia. E' inoltre iscritta nell'elenco degli Avvocati Mediatori presso l'Ordine degli Avvocati di Reggio Emilia.
} 
La sottrazione internazionale dei minori rappresenta argomento alquanto complesso e di ampia portata giuridica: il presente contributo si limiterà all'esame della fattispecie dal punto di vista della sua relazione con la compromissione del diritto di visita che il genitore subisce a causa della sottrazione e della tutela del minore nel caso venga tradotto in altro paese, nonché dei rimedi ai quali si può appellare. Come noto, le Convenzioni Internazionali a tutela del minore e la nostra legislazione garantiscono, in caso di separazione di due genitori, il diritto in capo al minore alla bigenitorialità, ovvero di mantenere un rapporto equilibrato e costante con entrambi i suoi genitori anche in caso in cui gli stessi siano separati e vivano in paesi diversi, e, al contempo, quello in capo ai genitori stessi di potere esercitare sul minore la responsabilità genitoriale nonostante la distanza geografica che li separa.

Nella prassi quotidiana, anche grazie all'aumento delle coppie con elementi di estraneità, non è infrequente che, in caso di intervenuta crisi familiare, uno dei due genitori decida di trasferirsi all'estero portando con sé il minore.

Si ha sottrazione internazionale, dunque, quando chi esercita la responsabilità genitoriale sul minore lo conduce all'estero senza l'autorizzazione dell'altro genitore, sottraendolo al paese di residenza abituale, oppure quando, nonostante vi sia stata l'autorizzazione, il minore non viene più ricondotto nel paese dove è radicata e stabilizzata la sua residenza abituale.

Non si può, dunque, esaminare la fattispecie senza prima fornire la nozione di residenza abituale del minore.
Ricaviamo direttamente tale definizione dall'art. 3 della Convenzione dell'Aja del 25/10/1980 che fa riferimento al luogo dove il minore ha di fatto il centro dei proprio interessi e dei propri legami affettivi in virtù di una durevole e stabile permanenza nel posto ove si svolge la sua quotidiana vita di relazione.

L'individuazione della residenza abituale del minore, dunque, è di cruciale importanza per determinare la legittimità della richiesta di reimpatrio da parte del genitore e, pertanto, per individuare il luogo dal quale il minore non deve essere allontanato e, se ciò avvenga, dove deve essere riportato.

In primis, dunque, l'autorità adita dovrà verificare se sussisteva o meno un accordo tra $i$ genitori di trasferimento all'estero del nucleo familiare e, in caso di esito negativo, se si è in presenza dei presupposti che delineano un illecito trasferimento in base ai criteri di cui all'art 3 della Convenzione.

La Convenzione dell'Aja, dedicata per lo più agli aspetti civili della sottrazione, ovvero alle istanze di reimpatrio ed alla regolamentazione o ripristino del diritto di visita del genitore non affidatario, è, insieme alla Convenzione di Lussemburgo del 20/5/1980 sul riconoscimento e l'esecuzione delle decisioni in materia di affidamento e di ristabilimento dell'affidamento, la principale fonte utilizzabile per contrastare il fenomeno e risolvere le controversie derivanti dagli illeciti trasferimenti dei minori.

Per potere attingere, dunque, alla tutela offerta dalle suddette convenzioni, è preliminarmente necessario stabilire quando un trasferimento del 
minore, o il suo mancato rientro, sono da considerarsi illeciti.

L'art 3 della Convenzione dell'Aja stabilisce, a questo proposito, che il trasferimento è illecito: a) quando avviene in violazione dei diritti di custodia assegnati ad una persona istituzione o altro ente, congiuntamente o individualmente, in base alla legislazione dello Stato nel quale il minore aveva la sua residenza abituale immediatamente prima del trasferimento o del suo mancato rientro; b) quando tali diritti vanno effettivamente esercitati, congiuntamente o individualmente, al momento del trasferimento del minore o del suo mancato rientro, o avrebbero potuto esserlo se non si fossero verificate tali circostanze

Ciò detto, ai fini della applicabilità delle garanzie offerte dalla Convenzione citata, occorre che il diritto di affidamento o di visita violato si fondino sulla legge o su una decisione giurisdizionale o amministrativa dello stato di residenza abituale del minore prima della sottrazione e che tale diritto sia stato effettivamente esercitato dal genitore che ha subito la sottrazione prima che questa fosse messa in atto e non sia sia stato prestato consenso, anche successivo, all'espatrio del minore.

A tali presupposti, si dovranno aggiungere l'eta inferiore ai sedici anni del minore tradotto, il fatto che non sia trascorso più di un anno dal momento della sottrazione e che dalla restituzione non derivi alcun danno morale e materiale al minore.

Dovrà altresì tenersi conto dell'opposizione al rientro manifestata dal minore stesso.

Da quanto fino ad ora esposto, si deduce che occorre distinguere la fattispecie della sottrazione internazionale del minore dal trasferimento all'estero del genitore in presenza di determinate circostanze dettate dal giudice in punto all'affidamento.

Un esempio pratico soccorre a chiarire perfettamente la suddetta distinzione: la Cassazione Penale, con sentenza n. 31717 del 6/6/2008, ha assolto una madre, che si era trasferita all'estro con il figlio, dal reato di cui all'art 388 c.p. e di quello di sottrazione internazionale di minore.

Alla madre, infatti, in virtù di provvedimento giudiziario, era stato concesso l'affidamento esclusivo del figlio. Poiché la legge n. 64/1994, che ha reso esecutiva in Italia la convenzione dell'Aja, attribuisce al genitore affidatario il diritto di stabilire la propria residenza con il minore all'estero, la madre non ha posto in essere una sottrazione illecita. Con la conseguenza che il genitore titolare del diritto di visita nei confronti del figlio non è legittimato a chiedere il rientro immediato del bambino ai sensi dell'art 8 della Convenzione dell'Aja (vd anche Cass. Civ. n. 8481 del 4.4.2007) .

In altre parole, il genitore non affidatario gode di una tutela in forma affievolita, avendo il solo diritto di agire affinché gli sia garantito il suo effettivo diritto di visita nei confronti del figlio attraverso una redefinizione delle relative modalità.

In quest'ultimo caso, il foro competente sarà quello della residenza abituale del figlio in virtù dell'art 8 del regolamento 2201/2003 che sancisce il principio del rapporto di prossimità del minore al giudice che deve decidere in ordine alle modalità di visita del bimbo stesso nei confronti del genitore non collocatario o non affidatario. 
In altre parole, la Convenzione, all'art 5, distingue in modo netto il diritto di affidamento da quello di visita, definendosi il primo come il potere comprensivo di tutti i diritti concernenti la cura della persona del minore ed il secondo come il diritto di condurre il minore in un luogo diverso dalla sua residenza abituale per un periodo limitato di tempo.

Abbiamo dunque appurato che per aversi illecita sottrazione occorre [art 3 Convenzione dell'Aja lettera a)] sia posta in atto la violazione della custodia e del diritto di affidamento.

$E^{\prime}$ quindi fondamentale, per capire la portata della norma e contestualizzarla, chiarire il concetto di custodia e di effettivo esercizio dell'affidamento: tali nozioni, secondo una interpretazione internazionalmente e convenzionalmente orientata, prescindono dalla sussistenza di un titolo giuridico, concretizzandosi in una situazione di fatto che si esplica nella cura morale e materiale del minore. La verifica della sussistenza o meno della violazione andrà poi ancorata all'esame della circostanza che il diritto di custodia da parte del genitore fosse effettivamente esercitato al momento del trasferimento del minore.

Da ciò consegue che, nonostante il regime dell'affido condiviso implichi che la scelta della residenza del minore debba essere assunta congiuntamente in accordo tra $i$ genitori, potrà tuttavia essere considerato lecito un trasferimento unilaterale del minore all'estero laddove il genitore dimostri che, di fatto, l'altro non esercitava il diritto/dovere di custodia e di affidamento (vd per tutte Cass. Civ. n. 12293 del 19/5/2010)

Quali, dunque, i rimedi a disposizione del genitore al quale il minore è sottratto illecitamente a causa del suo trasferimento all'estero ad opera dell'altro?

Egli dovrà adire l'Autorità Centrale presso il Ministero della Giustizia per ottenere il provvedimento di ordine di reimpatrio del minore. A questo proposito, l'art 12 della Convenzione dell'Aja subordina la presentazione dell'istanza al fatto che sia trascorso un periodo inferiore ad un anno a decorrere dal trasferimento o dal mancato rientro ed alla circostanza che il minore non abbia ancora compito i 16 anni.

Quando, invece, ad essere violato è il diritto di visita, perché l'altro genitore si è trasferito all'estero con il minore in virtù di un provvedimento di affidamento esclusivo a suo favore, il genitore potrà presentare una istanza all'Autorità Centrale dello Stato in cui è stato condotto o trattenuto il minore finalizzata all'organizzazione $\mathrm{O}$ alla tutela dell'esercizio effettivo del diritto di visita in ragione del mutato assetto della collocazione del minore ai sensi e per gli effetti dell'art 21 della Convenzione dell'Aja.

In questo caso, le Autorità Centrali sono obbligate ad assicurare un pacifico esercizio del diritto di visita e ad assolvere ad ogni condizione cui l'esercizio di tale diritto possa essere soggetto, oltre che a rimuovere, per quanto possibile, ogni ostacolo all'esercizio di tali diritti. A questo proposito, è interessante un cenno alla interpretazione che, negli ultimi anni, la Corte Europea dei Diritti dell'Uomo (CEDU) ha fornito della Convenzione dell'Aja.

In tema di ritorno del minore in patria, infatti, la CEDU ha imposto alle autorità nazionali l'obbligo di effettuare un esame approfondito della situazione della famiglia del bambino 
attraverso una valutazione di tutti gli aspetti psicologici, materiali e medici conseguenti al ritorno del minore affinché tale reimpatrio non possa poi avere effetti pregiudizievoli o dannosi sul minore stesso.

Ne consegue la condanna da parte della Corte di Strasburgo nei confronti delle autorità nazionali che adottino un provvedimento di reimpatrio in modo automatico e preconfezionato, senza che su tutto prevalga il supremo interesse del minore.

Citiamo, tra tutti, il caso Neulinger Shuruk contro Svizzera deciso dalla Corte dei Diritti dell'Uomo con sentenza n. 41615/07: su ricorso di un padre, l' Autorità Israeliana aveva disposto il ritorno in Israele di un minore trasferito dalla madre in Svizzera. Quest'ultima, sostenendo di avere agito nel preminente interesse del figlio per liberarlo dalle regole ferree, rigide e dagli obblighi religiosi cui il padre voleva sottoporlo, otteneva che la Corte giudicasse inopportuno il rientro del minore in Israele.

Oltre al supremo interesse del minore, vi è un altro aspetto che deve essere considerato e che, talvolta, può contribuire ad escludere la fattispecie di sottrazione internazionale, superando le stesse disposizioni del Giudici, ovvero la volontà del minore.

Un esempio chiarirà l'assunto: in un giudizio di divorzio, il giudice tedesco affidava la figlia al padre. Ma la madre conduceva e trasferiva la figlia in Italia. Il padre chiedeva e otteneva, dunque, dal Tribunale per i Minorenni di Palermo l'immediato rientro della bambina in Germania, valutato il suo interesse al ripristino della situazione di affidamento precedente al suo illecito trasferimento.

La peculiarità del decreto del Tribunale consisteva nel non avere dato rilevanza alla manifestazione di volontà della bambina di rimanere con la madre in Italia, in quanto non ritenuta sufficientemente dotata di capacità di discernimento.

L'esito del provvedimento de quo viene ribaltato dalla Corte di Cassazione, adita dalla madre che, con sentenza n. 14561 del 30/5/2014, ha ritenuto grave il fatto di avere il giudice di prime cure trascurato la manifesta volontà della figlia, di 13 anni, di volere rimanere in Italia con la madre.

La decisione della Suprema Corte appare conforme con quella interpretazione del combinato disposto degli artt 12 e 13 secondo comma della Convenzione che legittima il Giudice a negare il reimpatrio se il minore vi si oppone ed ha una età ed una capacità di discernimento che rendono opportuna la considerazione del suo parere ai fini decisori.

\section{Riferimenti bibliografici.}

- Carbone S. M., Queirolo I. (a cura di), Diritto di famiglia e Unione Europea, Giappichelli, Torino, 2008.

- Contiero G., L’affidamento dei minori: condiviso, esclusivo, a terzo, diritto di visita dei nonni, affidamento e sottrazione internazionale dei minori, Giuffrè, Milano, 2009.

- Preziosi S. (a cura di), Delitti contro la famiglia, Edizioni Scientifiche Italiane, Napoli-Roma, 2011.

- Sesta M., Arceri A. (a cura di), L’affidamento dei figli nella crisi della famiglia, UTET giuridica, Torino, 2012. 